\title{
Advanced industrial laser systems and applications
}

B. Schmidt, M. Schaefer

B. Schmidt, M. Schaefer, "Advanced industrial laser systems and applications," Proc. SPIE 10525, High-Power Laser Materials Processing: Applications, Diagnostics, and Systems VII, 1052502 (15 February 2018); doi: 10.1117/12.2299534

SPIE. Event: SPIE LASE, 2018, San Francisco, California, United States 


\title{
Advanced industrial laser systems and applications
}

\author{
B. Schmidt*a ${ }^{\mathrm{a}}$ M. Schaefer ${ }^{\mathrm{b}}$ \\ ${ }^{a}$ TRUMPF Photonics, Inc., 2601 US Route 130 Sout, 08512 Princeton, NJ USA \\ ${ }^{\mathrm{b}}$ TRUMPF Lasertechnik GmbH, Johann-Maus-Str. 2, 71254 Ditzingen, Germany
}

\begin{abstract}
Industrial laser systems continue to evolve in terms of power levels, brightness and mode of operation. While continouswave (cw) laser systems drive to more efficiency, compactness and robustness, ultra-short pulse (USP) solid-state lasers (SSL) gain increasing attention from industry due to their higher average power levels, increasing pulse energies and more flexible repetition rates, eventually enabling increased productivity and novel materials processing applications. TRUPMF's laser systems portfolio starting in 1985 mirrors this general development and is therefore an interesting subject to reflect the path of industrial laser systems from early $\mathrm{CO}_{2}$ lasers to the latest generation of diode pumped nanosecond-pulsed disk lasers with up to $400 \mathrm{~W}$ of average output power at $343 \mathrm{~nm}$. Today, our advanced laser families enable novel applications such as EUV light generation, laser lift-off and surface annealing.
\end{abstract}

Keywords: Disk laser, fiber laser, diode laser, ultra-short pulsed laser, welding, cutting, annealing

\section{INTRODUCTION}

Laser applications in industry and the manufacturing of respective laser systems have rapidly evolved during the past three decades. TRUMPF's strategy in this field is a strong vertical integration of core technologies, an approach we share with major manufacturing companies of industrial laser systems. Incorporating central technologies such as $\mathrm{CO}_{2^{-}}$, Disk-, Fiber-, Slab- and Direct Diode Lasers gives TRUMPF the full bandwidth to serve most prominent applications in the field of laser based processing. This development is accompanied by new industrial grade advances in beam guiding and shaping, as e.g. the flexible transport of ultra-short laser pulses by hollow core fibers or modular beam guiding optics for large area rapid thermal processing. Furthermore, advanced sensors in laser systems and in beam guiding optics (e.g. OCT) combined with advanced data networks and specific algorithms allow in situ condition and process monitoring. This provides new insights in laser-matter interaction parameters and gives rise to process developments by means of pure machine learning methods, midterm.

On the one hand high-quality cutting edges (no burr) can be achieved by means of BrightLine which is available for $\mathrm{CO}_{2}$ lasers and solid-state lasers. On the other hand improving the design of the cutting nozzle boosts the feed rate by up to 100 percent for solid-state laser machines that employ fusion cutting with nitrogen while the new Highspeed Eco cutting process requires $70 \%$ less nitrogen in average. With this as a baseline it is already possible to attain applications like spatter-reduced cw- and pulsed laser welding of batteries, welding of transparent materials utilizing ultra-short laser pulses, annealing of amorphous silicon as well as more exotic tasks such as the active triggering of lightning during a thunderstorm.

\section{ONGOING CHANGE IN INDUSTRIAL LASER TECHNOLOGY}

\section{$2.1 \mathrm{CO}_{2}$ lasers}

At TRUMPF $\mathrm{CO}_{2}$ lasers have always been seen as a tool for material processing integrated in machine tools e.g. by combining punching and laser cutting. However, in the early 80 s no robust industrial-grade lasers were available with *berthold.schmidt@us.trumpf.com; phone 1609480 2047; TRUMPF.com 
sufficient beam quality at satisfying power levels for $\mathrm{cw}$ laser applications. In addition, potential suppliers had not been able to reach 1000 Watt, a power level the market requested by that time. Therefore, TRUMPF started to make its own $\mathrm{CO}_{2}$ laser at the beginning of the 1980s. Consequently, TRUMPF showcased itself as a laser manufacturer with the first self-developed and produced $\mathrm{CO}_{2}$ laser in 1985 (see left picture in Figure 1). The latter had an output power of over $1 \mathrm{~kW}$ and was the first compact commercial laser resonator featuring radio-frequency excitation (RF excitation). ${ }^{1}$
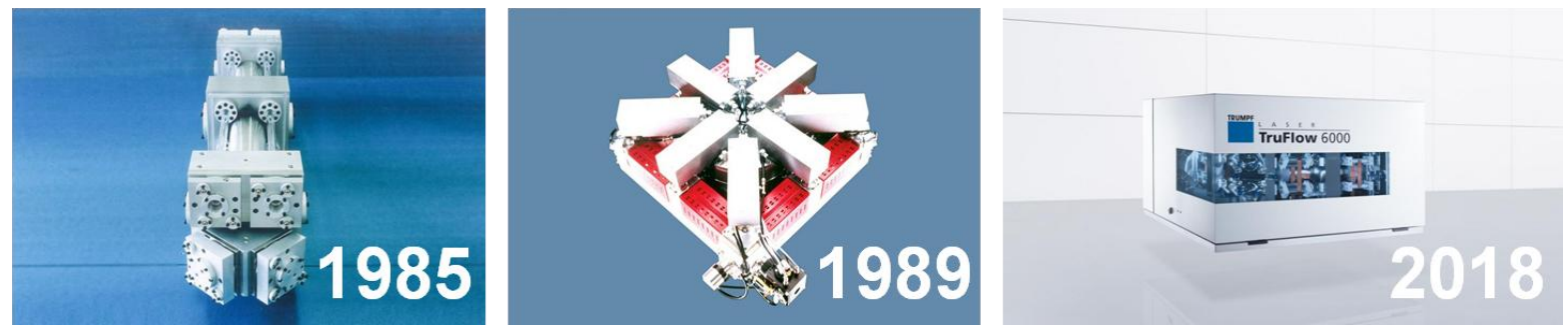

Figure 1. $\mathrm{CO}_{2}$ laser development at TRUMPF since 1985 with the TRUMPF TLF 1000 (left) and subsequent TruFlow folded cavity laser generations (middle and right). ${ }^{1}$

In 1989 at the EMO (Exposition Mondiale de la Machine Outil, the world leading trade show for metal working) in Hanover, the first "folded" laser was presented. Until 2009 more than 20.000 TruFlow systems were shipped worldwide. These lasers were mostly used for laser cutting of sheet metal made of steel, stainless steel, aluminum and further metal alloys. Cutting of plates up to $25 \mathrm{~mm}$ mild steel suddenly got feasible. Moreover, laser welding tasks arise - mainly for thick sheet metals like in the ship building industry. ${ }^{2}$

$\mathrm{CO}_{2}$ lasers, although being considered "old-fashion" in laser metal applications, are a good example to express TRUMPF's long-term vision and entrepreneurial spirit. Based on the existing high-power $\mathrm{CO}_{2}$-laser technology TRUMPF was able to develop a $30 \mathrm{~kW}$ master-oscillator power-amplifier (MOPA) system, which serves as a drive laser for high-harmonic generation in tin (Sn) droplets in future extreme ultraviolet (EUV) systems for microlithography. This approach currently represents the most promising path to produce next generation of microchips whose processing speed can hold up with Moore's law. ${ }^{3}$ In this project the aim is to generate extreme ultraviolet (EUV) light, with a wavelength of $13.5 \mathrm{~nm}$ (see Figure 2).

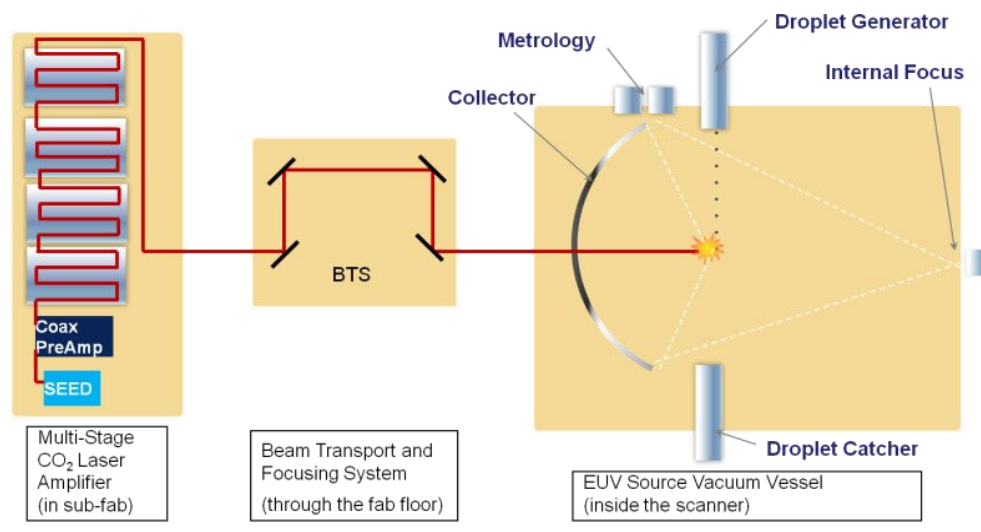

Figure 2. EUV emission and tin mitigation process concept. ${ }^{4}$

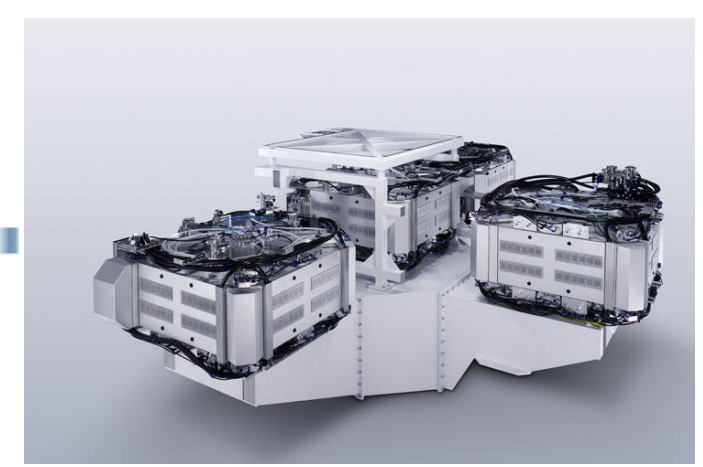

Figure 3. $\mathrm{CO}_{2}$ drive laser for a $13.5 \mathrm{~nm}$ EUV lithography system (3 Stage Amplifier)

The key component for these highly complex manufacturing techniques is the $\mathrm{CO}_{2}$ amplifier system that generates laser pulses providing a pulse duration of about $100 \mathrm{~ns}$ at a pulse repetition rate of $50 \mathrm{kHz}$ with $\sim 30 \mathrm{~kW}$ of average output power, according to a pulse energy of $600 \mathrm{~mJ}$ and a pulse power of $6 \mathrm{MW}$. In Figure 2 the concept of tin (Sn) mitigation and emission of EUV light generation is depicted. First, a $\mathrm{CO}_{2}$ pre-pulse laser hits the $\mathrm{Sn}$ droplet and transforms it into a 
fine mist or a pancake-like shape respectively. Now, increased in size, the main laser pulse ionizes the mist which is converted into a high-temperature plasma $\left(\mathrm{T}_{\text {plasma }} \approx 500.000 \mathrm{~K}\right.$ ) that irradiates extreme ultraviolet light with a wavelength of $13.5 \mathrm{~nm}$ during recombination. ${ }^{3}$ To attain the laser beam intensity requested for the plasma process an almost diffraction-limited beam quality is required for the $\mathrm{CO}_{2}$ laser. ${ }^{4}$

\section{$2.2 \mathrm{CW}$ solid-state lasers (SSL)}

Although $\mathrm{CO}_{2}$ lasers continue to play an important role in advanced industries, developments in SSL brought new systems, such as disk- and fiber lasers to the market. These systems yield a shorter emission wavelength ( 1 um), thus having a higher absorption in metals combined with a significant higher system wall-plug efficiency. In addition, the absorption in the plasma plume above the (metal) work piece is highly reduced at $1 \mu \mathrm{m}$ compared to $\sim 10 \mu \mathrm{m}$, further enhancing the cutting process. After industrial grade SSLs with fiber beam delivery became available in the new millennium, a continuous shift in the the conventional market for laser metal applications took place which has impacted the industry during the past 15 years. Today, SSLs are the dominant light source for material processing. In Figure 4 the change in market share of $\mathrm{CO}_{2}$, solid-state lasers and direct diode lasers during the past years is depicted on a percentage basis.

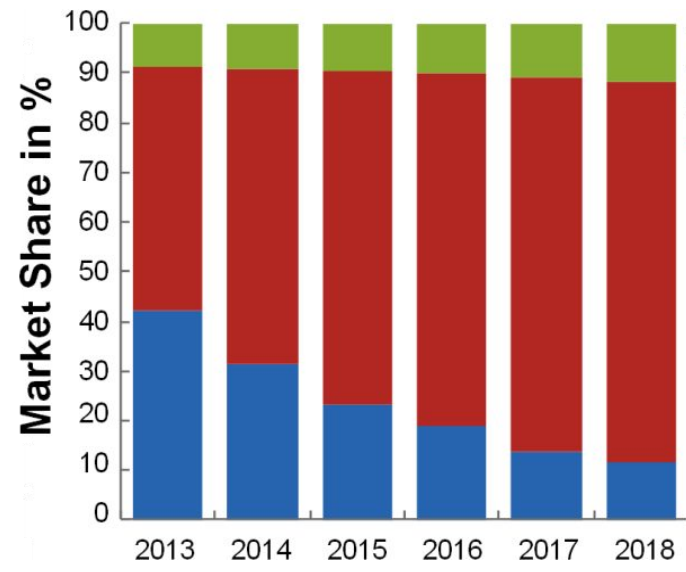

Systems with DDL

- Systems with $\mathrm{CO} 2$ lasers

- Systems with SSL

(incl. USP and CW lasers)

Figure 4. Market share of different laser technologies during the past four years and forecasted for 2018. Original data derived from ${ }^{5}$.

TRUMPF has always been actively involved in conducting this technical change in the laser industry. Accompanied by only a few remaining laser manufacturers, TRUMPF nowadays offers a full bandwidth of CW and USP solid-state highpower lasers for high-tech industrial production. While lasers become more and more versatile a continuously growing industrial community incorporates, accepts or still explores this tool as a central production solution for a wide range of dedicated markets such as e-mobility, portable devices, semiconductor manufacturing, car and ship building, construction and scientific applications.

\subsubsection{Disk laser platform}

Compared to some other companies TRUMPF does not believe there is a single laser technology that can serve most applications. In reality, best in class quality for each laser material process requires optimum parameters, which consequently define the specifications of the applied laser source. That is the reason why TRUMPF is offering "the power of choice" to the market: a balanced SSL technology portfolio of direct diode lasers, fiber lasers, slab lasers and thin-disk lasers. It is already foreseeable that the latter due to its high versatility will remain a central building block in TRUMPF's laser portfolio, long term. Disk lasers were first introduced by TRUMPF as a commercial product in 1999. Since an efficiently cooled $\mathrm{Yb}$-doped thin-disk is used as active medium, the laser yields strongly reduced thermal aberrations and thermo-mechanical stress for highest beam qualities even at elevated output powers. ${ }^{6}$ In comparison to fiber lasers the large diameter of the pump spot on the disk allow pumping with low brightness diodes ( $450 \mathrm{~mm} \cdot \mathrm{mrad})$. 
Therefore the Disk laser is a perfect brightness converter which becomes a significant cost advantage when power levels of multiple kWs at high brightness are required.

One of the latest technological developments in CW high power disk lasers is the new generation of TRUMPF's TruDisk product line offering up to $6 \mathrm{~kW}$ output power from a single disk at a wavelength of $1030 \mathrm{~nm}$ with high beam quality of up to $4 \mathrm{~mm} \cdot \mathrm{mrad}$ (Figure 5 ). ${ }^{7}$ This device comes along with a footprint, reduced by $50 \%$ compared to previous models. TRUMPF was eager to provide an industrially even more robust laser device with an innovative and space-saving cooling architecture (Supply temperature of $5-38^{\circ} \mathrm{C}$ ) as well as significantly increased system efficiency e.g. by means of a complete reduction of the diode current to $0 \mathrm{~A}$ in between two processing steps (cycle time: $\sim 100 \mu \mathrm{s}$ ). As Industry 4.0 readiness is a central driver in our system design considerations, the new generation of TruDisk lasers features optimum hardware and time-synchronized data recording of all sensors, providing high-quality data for virtual analyzes enabling services such as condition monitoring and predictive maintenance. This enables our customers to continuously optimize and analyze its processes and allows TRUMPF to support those efforts with condition monitoring of the laser based on sophisticated algorithms analyzing the $\sim 500$ different signals of input data, modern laser systems generate e.g. during a cutting or welding process. ${ }^{8}$
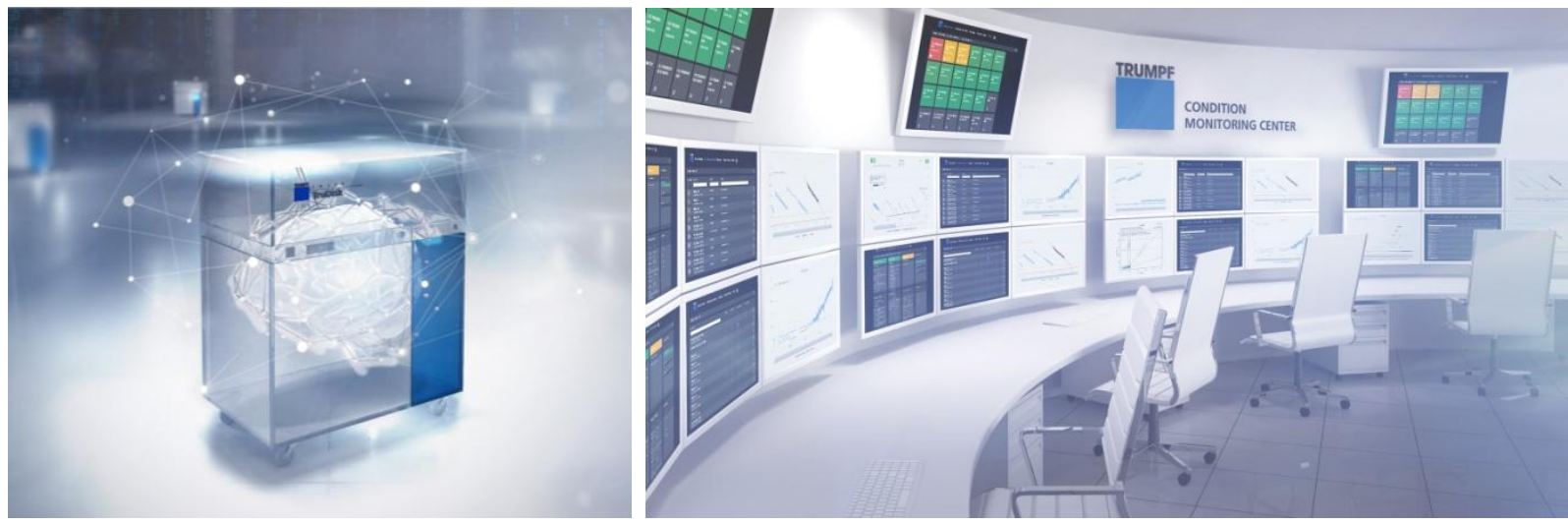

Figure 5. The new, smart TruDisk laser with an Industry 4.0 ready sensor platform utilizing big data algorithm in TRUMPF's condition monitoring center.

The high flexibility provided by the disk laser platform enables a wide variety of beam shaping and control features such as the new BrightLine Weld for example. During laser welding at a range of 6 to $20 \mathrm{~m} / \mathrm{min}$ welding speed severe spattering out of the weld pool normally occurs. The new approach allows high-quality weld seams while preventing this spatter induced mass loss at high welding speeds. Due to the patented dual-clad fiber, a completely flexible superposition of two formed laser beams out of one single laser source can be achieved with a fully adjustable power ratio. By means of this approach for the beam shaping, an intensity profile of the superimposed beams can be attained on the workpiece which is optimized for the welding task. ${ }^{8,9}$

\subsubsection{Fiber and direct diode laser}

In close cooperation with SPI as an affiliated entity, TRUMPF's fiber laser portfolio continues to expand incorporating a highly integrated value chain supported by a network of strategic suppliers. Since fiber laser offer nearly diffraction limited beam quality at $2 \mathrm{~kW}$ optical $\mathrm{cw}$ output power together with high wall-plug efficiency, they are opening new fields of applications which are of increasing interest for the industry. Key for their success is that they can be offered not only at low cost, but with high system quality and sufficient robustness for a wide range of industrial processes. Aside from various $\mathrm{cw}$ - fiber lasers of multiple kWs, SPI also offers a wide variety of ns-pulsed fiber lasers with high repetition rate which are in particular of interest in the markets for e-mobility, laser marking and mobile devices. ${ }^{10}$ 

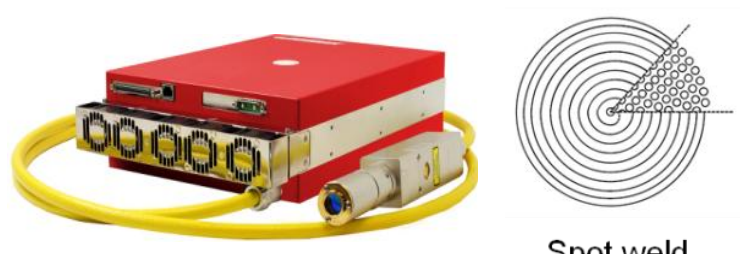

Spot weld
Al flow to surface

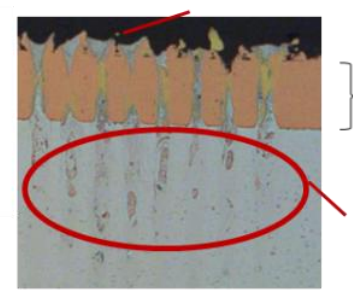

$150 \mu \mathrm{m}$

Cu in pool of solidification

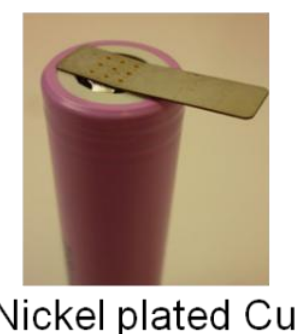

Figure 6. SPI's redENERGY pulsed fiber laser with up to $200 \mathrm{~W}$ average power and a key application of welding dissimilar materials.

Another exiting laser systems technology are direct diode lasers offering highest energy efficiencies as the semiconductor laser diodes are combined without any further optical-optical power conversion. Nowadays, TRUMPF offers multi-kW class direct diode lasers of highest wall-plug efficiency incorporating spatial, polarization and coarse wavelengths beam combining. These TruDiode lasers with a BPP of 30 to $50 \mathrm{~mm} \cdot \mathrm{mrad}$ and up to $6 \mathrm{~kW}$ output power are the perfect production solution for applications requiring lower brightness, such as welding, brazing and laser metal deposition for instance. To improve the BPP of direct diode lasers towards high-brightness applications, TRUMPF has intensively looked into a novel beam combining scheme in the past five years, called dense wavelength multiplexing (DWM). DWM lasers incorporate techniques where hundreds of semiconductor laser diodes are wavelength stabilized, of which each is operating at a slight different wavelength, and beam-combined within a common external feedback cavity. The cavity itself is setup as a closed-loop feedback system that provides in the forward direction dense spectral beam combining and in the backwards direction narrow-band feedback to stabilize each individual diode emitter. Since neighboring laser diodes can be superimposed into a single beam, significantly enhanced power levels can be achieved at high brightness. Whereat, the beam quality of the combined beam is mainly determined by the beam quality of the individual emitter. ${ }^{11}$

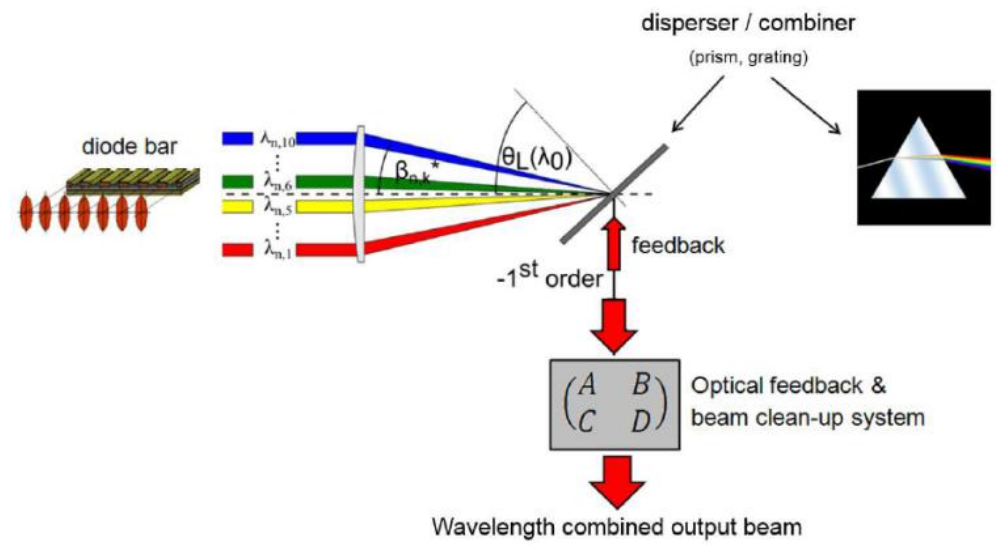

Figure 7. Sketch of TRUMPF's DWM diode laser. ${ }^{11}$

The resulting laser systems come with fiber beam delivery and are suitable for fine welding and remote scanner welding as well as cutting applications that were not addressable by conventional direct diode lasers incorporating spatial, polarization and coarse wavelengths beam combining up to date. DWM lasers combine high wall plug efficiency of $>40 \%$ (including electronics and cooler) with excellent beam quality of $5 \mathrm{~mm} \cdot \mathrm{mrad}$ delivering $\mathrm{kW}$ 's of optical power out of a $150 \mu \mathrm{m}$ fiber in a very compact cabinet with a footprint of only $0.57 \mathrm{~m}^{2} .^{11}$ Because of its vertical integration, TRUMPF is able to implement high-brightness laser diodes for the system on the same high-volume diode laser platform that is a central building block in the TruDisk systems and therefore guarantees high quality coming along with the highvolume production capability. 
The system comes along with the typical functionalities of TRUMPF lasers such as power sharing and switching, plugand-play fiber exchange, online power control, energy saving modes, an integrated cooling system and an advanced control system including industry 4.0 capabilities. With a new generation of highly efficient and compact DWM lasers, TRUMPF will set a new benchmark promoting high brightness direct diode technology for industrial metal processing applications. $^{11}$

\section{LASER SYSTEMS FOR SHORT PULSED AND ULTRA-SHORT PULSED APPLICATIONS}

\subsection{TruMicro series}

TRUMPF's portfolio incorporates a broad bandwidth of SSL technologies when short pulse and ultra short pulse laser systems at ns, ps and even fs pulse lengths are required. At lower energy levels fiber lasers such as the TruMicro 2000 series ( 20 Watts at maximum $50 \mu \mathrm{J}$ with pulse durations of $<400 \mathrm{fs})^{12}$ and the already mentioned redENERGY laser from SPI play an important role in industry and for scientific applications. When high average power levels and high pulse energies are demanded thin-disk amplifiers, due to their superior properties, are the perfect building block for various short pulse TruMicro laser systems. Straight forward, a thin-disk short pulse laser can be laid out e.g. to operate in a so called cavity-dumped configuration, where the pulse duration of a few 10ns equals the round trip time of the laser cavity. Cavity-dumped disk lasers (base for TRUMPF's TruMicro 7000 series) take advantage of the enormous high energy that can be stored within the intracavity resonator operating at gain saturation as shown in Figure $8 .{ }^{13}$ The basic idea of cavity dumping is to switch the transmission of the output coupler of the laser cavity. When switching the polarization with a Pockels cell the stored intracavity energy is released (dumped) yielding average IR powers as high as $4 \mathrm{~kW}$ with repetition rates in the $\mathrm{kHz}$ regime. ${ }^{13}$

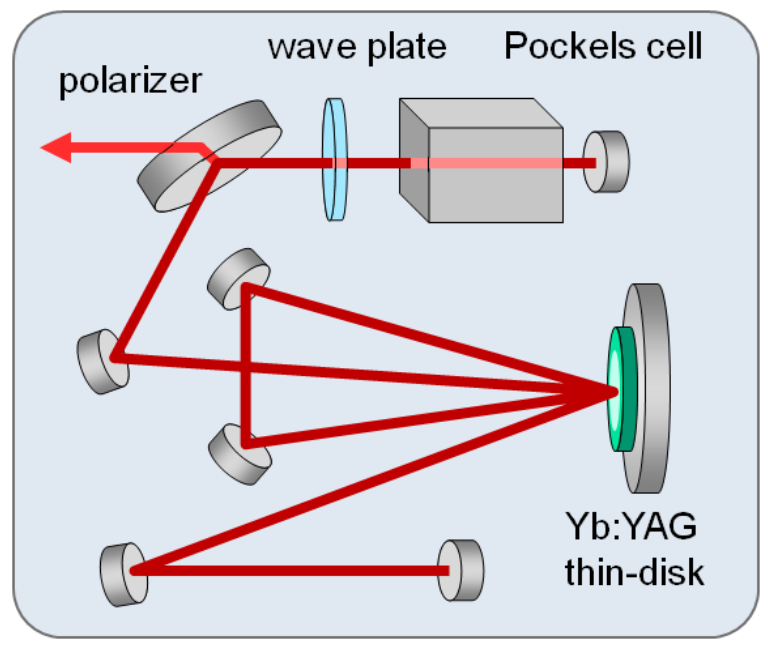

Figure 8. Thin-disk laser principle with „,cavity dumping“.

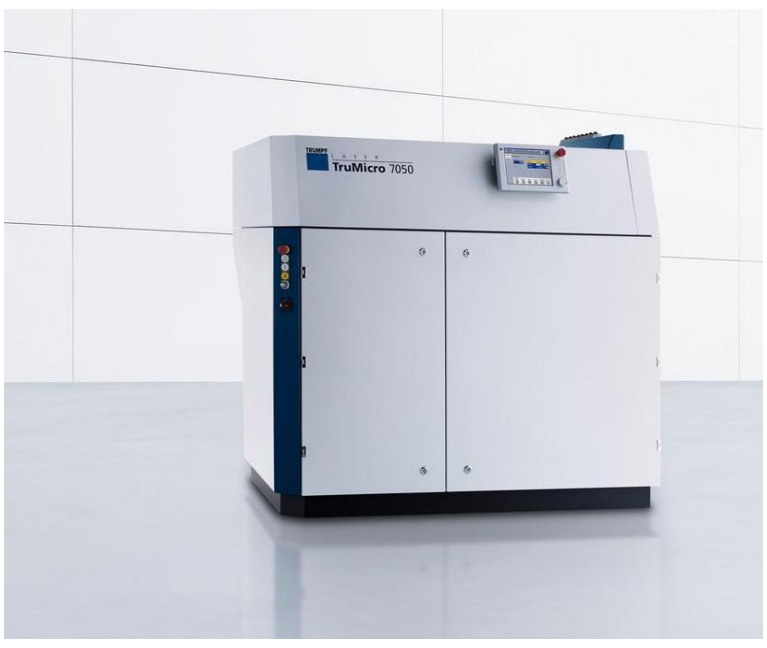

Figure 9. TruMicro 7000 series

High-energetic and narrowband ns pulses in the infrared can be used to efficiently enable external-cavity frequency conversion such as second harmonic generation (SHG) and third harmonic generation (THG). The conversion towards the third harmonic can be done either in single stage or in order to increase the conversion efficiency in a cascaded way by splitting and overlapping the IR and SHG. For second harmonic generation $800 \mathrm{~W}$ of output power at $515 \mathrm{~nm}$ has been demonstrated ${ }^{13}$ whereas for the third harmonic generation an average power of above $500 \mathrm{~W}$ at a wavelength of $343 \mathrm{~nm}$ at $<20 \mathrm{~ns}$ and $10 \mathrm{kHz}$ has been achieved. ${ }^{14}$ High average power cavity-dumped thin-disk laser yield a broad range of important industrial applications such as drilling or creating hybrid metal-plastic connections, edge deletion of thin-film solar modules, and at elevated powers also laser-lift-off (LLO) for flexible displays and solid-state laser annealing (SLA) of amorphous silicon. 
Extending the principle of using the thin-disk as an amplifier is the base concept of TRUMPF's most prominent industrial grade USP laser type, the TruMicro 5000 series, where the thin-disk is utilized as a regenerative amplifier ("Regen"). USP disk laser gain more and more of importance due to their nearly diffraction limited beam properties and consequently their potential for applications such as precise drilling of metals without any heat affected zone ${ }^{15}$ and no need for post processing, advanced cutting and welding of transparent materials. ${ }^{16}$

In contrary to the cavity-dumped layout, a seed laser defines the pulse shape entering the thin-disk Regen, where it is caught, then amplified by $>5$ orders of magnitude, subsequently coupled out, and modulated externally. The incoupling and outcoupling of seed pulses into and out of this resonator is obtained by polarization switching using an intracavity Beta-barium-borate (BBO) Pockels cells in conjunction with a polarizing beam splitter (PBS). BBO Pockels cells have the advantage to operate at $\mathrm{kW}$ average power levels with negligible thermal lensing, due to very low absorption. ${ }^{15}$

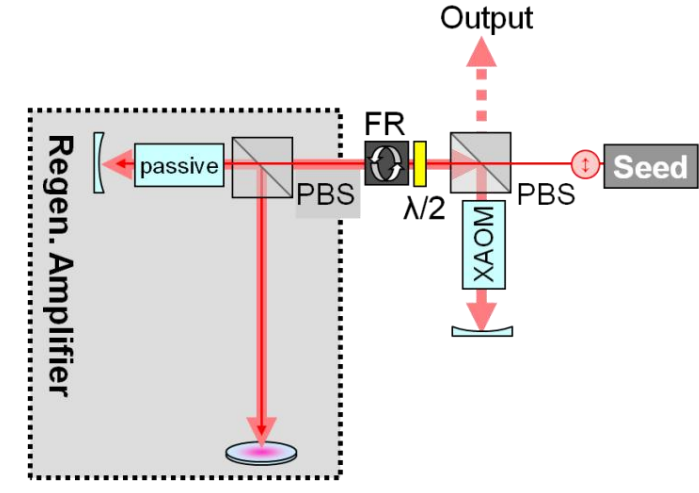

Figure 10. Schematic of a regenerative thin-disk amplifier.

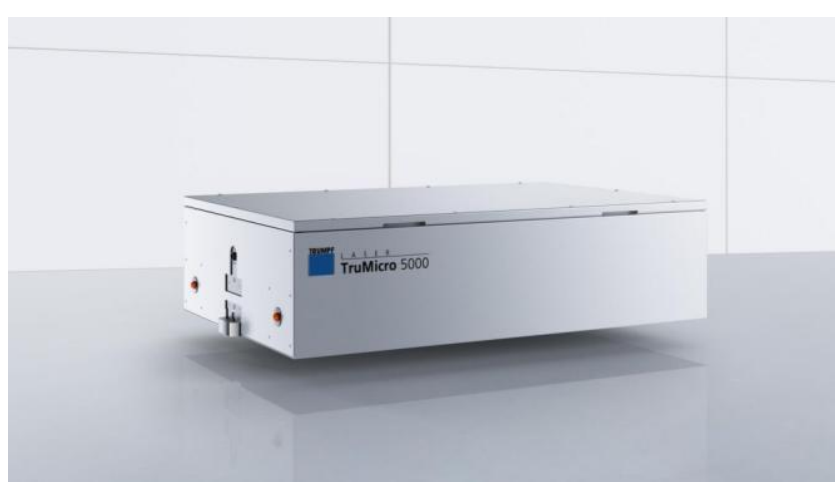

Figure 11. TruMicro 5000 series based on CPA delivering up to $150 \mathrm{~W}$ of $<10 \mathrm{ps}$ pulses at base repetition rates between $100 \mathrm{kHz}$ and $1 \mathrm{MHz}$.

The system, as depicted in Figure 11, contains a low power, mode-locked fiber seed oscillator, followed by the disk Regen, a telescope for precise adjustment of the output beam diameter, a highly efficient external acousto-optic modulator (XAOM) for precise and highly dynamic setting of the output energy, an optional, very compact grating based pulse compressor (TruMicro Femto Series incorporating chirped pulse amplification (CPA)), an optional frequency doubling or tripling module (to $515 \mathrm{~nm}$ resp. $343 \mathrm{~nm}$ ), and a mechanical safety shutter, as well as the required sensors, control boards, and drivers. ${ }^{15}$

\subsection{Short Pulse and Ultra-Short Pulse Power Scaling: Multi-Pass Cell}

Laser output powers from USP regenerative amplifiers or cavity dumped oscillators can be further enhanced, when combining those with a thin-disk multi-pass cell (MPC) for externally amplifying laser pulses. ${ }^{17}$ Depending on the configuration i.e. number of passes, amplification factors ranging from 3-25 can be realized. Since the MPC configuration is free of absorbing elements the B-Integral which is proportional to the optical intensity and the Kerr nonlinearity is highly reduced even at elevated pulse energies. Hence, the influence of self focusing and non-linear phase shift are severely alleviated, the MPC gives rise to highest amplification levels in industrial USP SSL systems. Furthermore, due to the linear amplification scheme without partially reflective outcoupling mirror no enhanced power load of the individual mirrors is present in the MPC.

As stated before, the MPC is highly flexible preventing almost all non-linear effects but enabling the amplification of pulses from ps over ns range of pulse duration even up to continuous wave power scaling. Therefore, this technique has high potential to be a versatile sub-unit within the TRUMPF's amplifier architecture enabling $>\mathrm{kW}$ power levels while having vanishing impact on the high beam quality. 


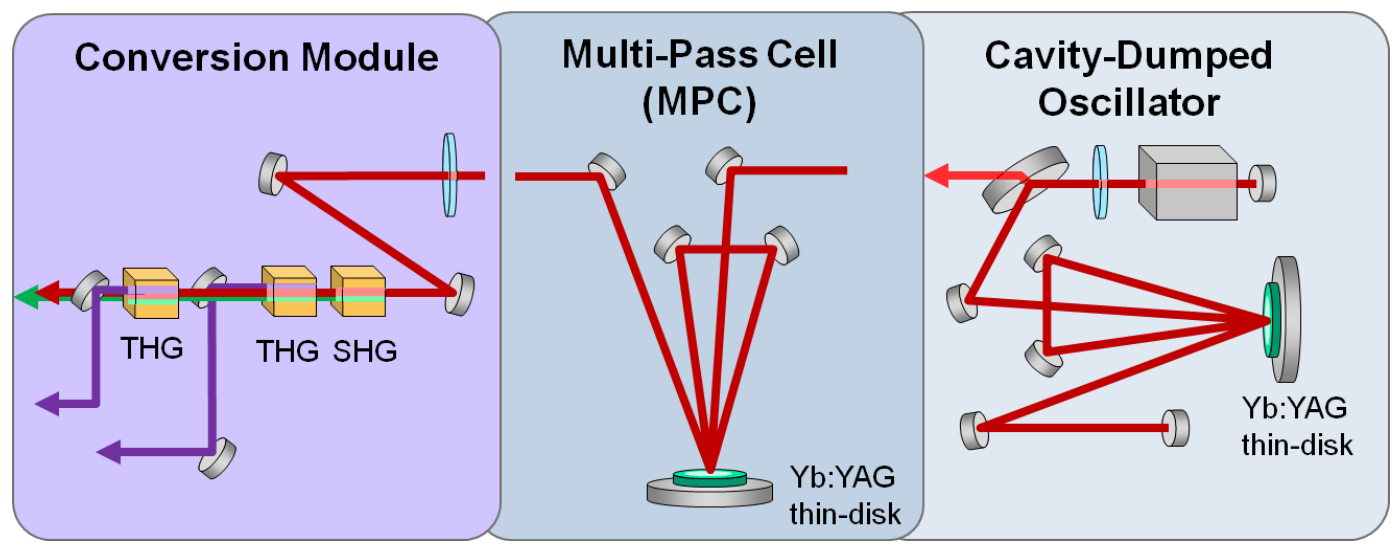

Figure 12. Cacity-dumped thin-disk laser principle with subsequent multi-pass cell and conversion module.

Some examples: Seeding a linear multi-pass amplifier system using a TruMicro 5000 with $100 \mathrm{~W}$ of average power at $1 \mathrm{ps}$ and $300 \mathrm{kHz}$, by 36 passes in a multi-pass cell an output power of $1.4 \mathrm{~kW}$ can be attained at same pulse duration $(\sim 1 \mathrm{ps}) .{ }^{17}$ Applying a TruMicro 7000 at $\sim 10 \mathrm{~ns}$ and $10 \mathrm{kHz}$ as seed laser, the multi-pass cell scales up the output power within 18 passes to $3 \mathrm{~kW}$. Such a combined system of a cavity-dumped Yb-YAG thin-disk laser with multi-pass cell and the optional subsequent conversion module is schematically depicted in Figure 12. As already mentioned, not only the power scaling for pulsed systems can be achieved but also for high-power cw laser systems like for the TruDisk platform. There, the amplification was performed in two identical stages, each consisting of an MPC in single-pass and an Yb-doped thin disk amplifier. A multimode TruDisk 6001 was used as seed laser providing $7 \mathrm{~kW}$ of $\mathrm{cw}$ power. Within two times 18 passes the system reached up to $20 \mathrm{~kW}$ output power at an optical-optical efficiency of $>60 \%$ and a minimum of power fluctuation without active stabilization of the MPC amplifiers as depicted in Figure 14 (here at $15.5 \mathrm{~kW}){ }^{18}$

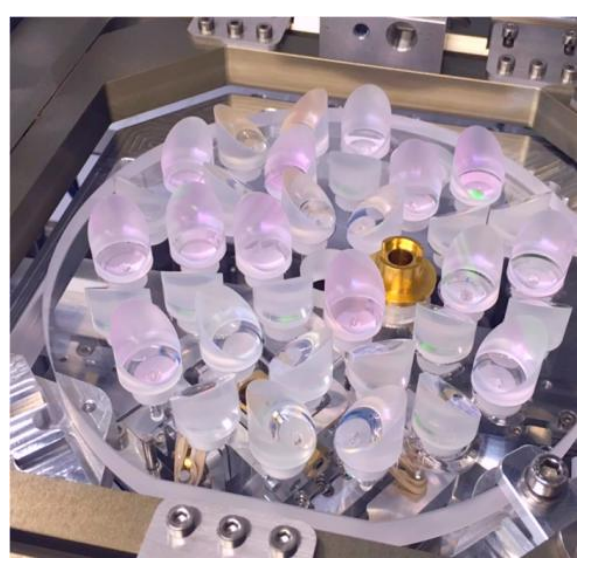

Figure 13. Monolithic mirror array of the multi-pass cell incorporating a laser welded lens configuration.

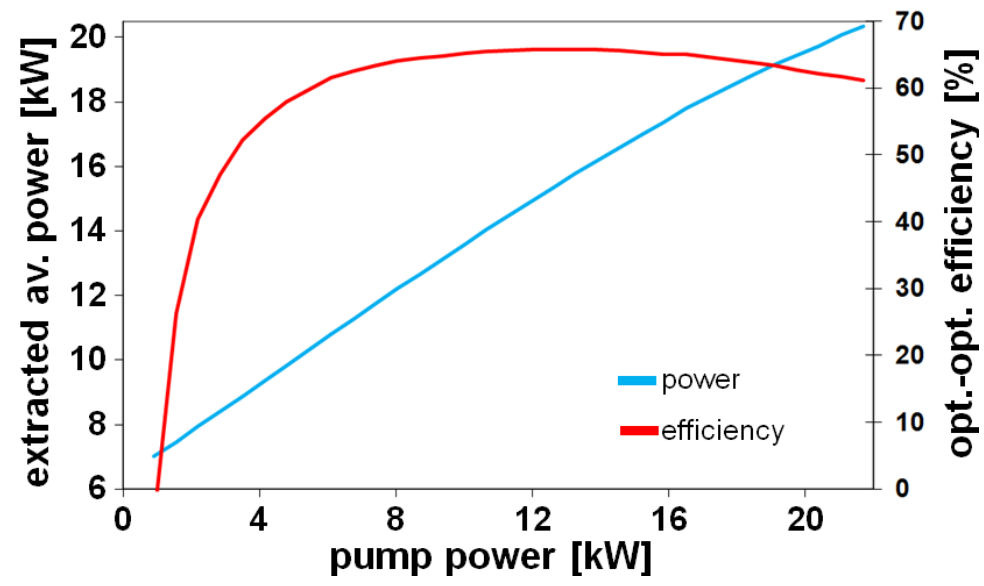

Figure 14. Output Power and optical to optical-efficiency of a TruDisk in combination with MPC.

Since May 2012 TRUMPF Scientific, a joint venture of the Max-Planck Institute of Quantum Optics and TRUMPF, explores and develops different kinds of exotic USP lasers for scientific and potential future industrial applications. The activities are manifold ranging from the development of ps lasers providing high repetition rate and high average output power to the generation of high-energetic, few-cycle fs pulses of extreme pulse peak powers. The targeted application are similar diverse and range from soft x-ray generation by means of inverse Compton scattering of ultra-short laser 
pulses for ultra-fast time resolved spectroscopy to EUV generation by means of higher harmonic generation induced by ultra high peak power fs pulses. Whereas ps pulses can in general be amplified in Yb:YAG regenerative and MPC amplifiers, the amplification of fs laser pulses of simultaneously high-average output power in Yb:YAG is restricted by the limited spectral gain bandwidth of the medium. At TRUMPF Scientific, fs laser pulses of high pulse energy and peak power are generated by using the scheme of optical parametric chirped pulse amplification (OPCPA). In this amplification scheme, low energetic fs pulses from a Ti:Sapphire laser are stretched to a pulse duration of a few ps, potentially pre-amplified in a regenerative amplifier, and eventually overlapped in a BBO crystal with a ps pulse from a pump disk laser (see Figure 15). ${ }^{19}$

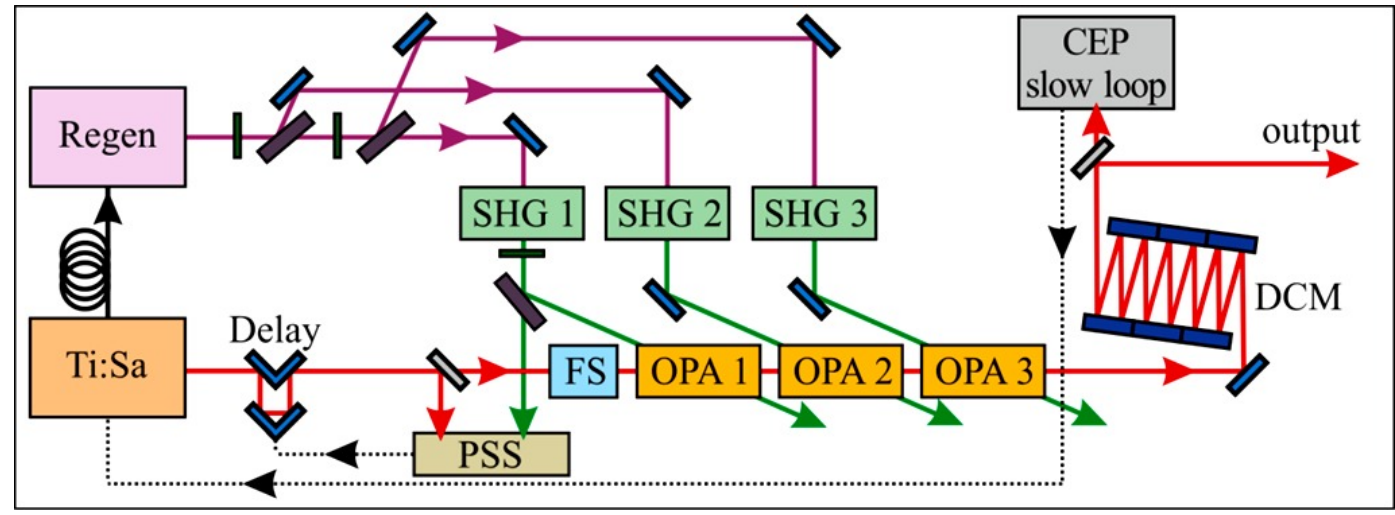

Figure 15. Schematic layout of the regenerative amplifier using optical chirped pulse amplification (OPCPA). Ti:Sa: Titaniumsapphire oscillator, Regen: regenerative amplifier, BS: beam splitter, WP: waveplate, TFP: thin-film polarizer, SHG: second harmonic generation, PSS: pump-seed synchronization, FS: fused silica stretcher, OPA: optical parametric amplifier, CEP slow loop: carrier-envelope phase slow-loop stabilization, DCM: double-chirped mirror compressor. ${ }^{19}$

In case of fulfilling phase matching conditions, one can drive the process of optical parametric amplification in the BBO crystal quite efficiently, without suffering the problem of spectral clipping as experienced in Yb:YAG at such pulse durations. Once the energy is transferred from the ps pump laser to the stretched fs laser pulse by the OPA process, which requires a precise temporal overlap of the pump and signal pulse in the BBO crystal, the amplified pulse can be recompressed in a grating compressor to its initial pulse duration of a couple fs. In summary, TRUMPF Scientifics lasers can be classified in ps and fs laser systems. The ps lasers range in pulse energy from a few $\mathrm{mJ}$ up to $200 \mathrm{~mJ}$, their amplification relies on regenerative amplifiers. Recently, we have demonstrated $1 \mathrm{~J}$ of pulse energy at repetition rate of $1 \mathrm{kHz}$ and a pulse duration of about 2 ps by post amplifying the output from a regenerative amplifier within a Yb:YAG MPC arrangement. ${ }^{20}$

Using the MPC for high ps amplification is of significant advantage due to the already mentioned reduction of the Bintegral preventing pulse shape deformation and lowering of peak intensities. Also, the risk of optical damage thresholds in the Pockels cells is overcome. With such a portfolio of different average output powers, pulse repetition rates and pulse energies (see Figure 16), we have the possibility to use these ps beam sources to pump and drive either single-stage or multi-stage OPCPA for the amplification of fs pulses. ${ }^{21} \mathrm{We}$ see that the schemes described above allow for pulses with shorter than $30 \mathrm{fs}$ at average output power levels of greater than $2 \mathrm{~kW}$, which are feasible for higher harmonic generation. Thus, $10^{17}$ to $10^{18} \mathrm{~W} / \mathrm{cm}^{2}$ become available with our laser sources for soft $\mathrm{x}$-ray and $\mathrm{k}$-alpha generation. $100 \mathrm{~W}$ of average output power with few mJ using OPCPA look feasible as well. 


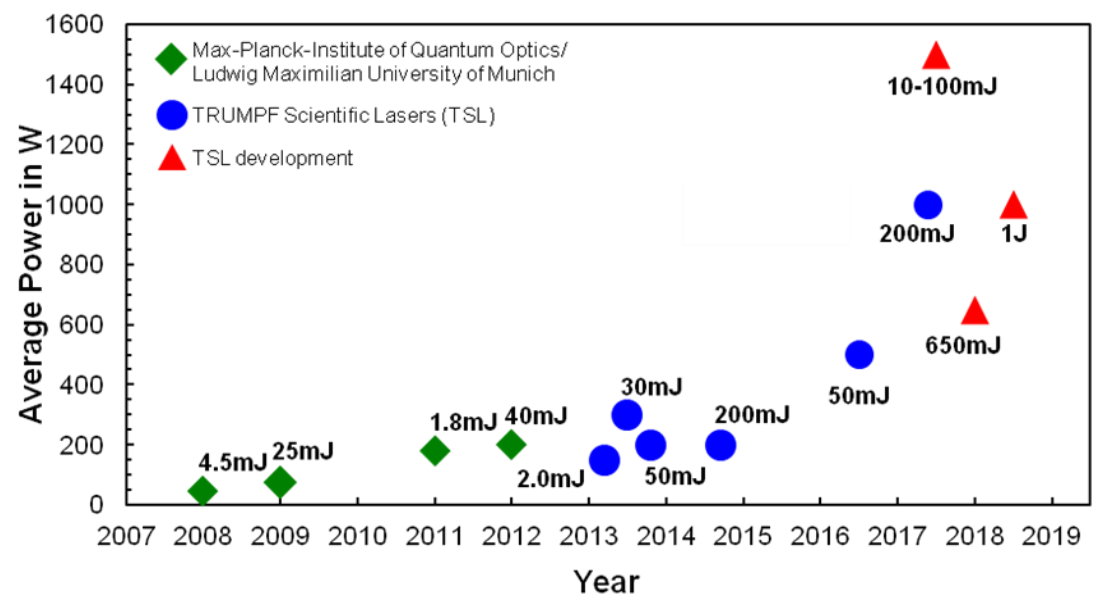

Figure 16. Development of high pulse and average power levels based on the thin-disk regen laser platform during the last 10 years (according to ${ }^{21}$ ).

\subsection{Beam shaping optics for advanced applications}

In addition to the optimization of various architectures of laser systems, it is central to the success in the industrial market to implement intelligent beam forming optics combined with smart sensors for in-situ diagnostics. Special laser beam shaping as enabler for new application is a field of extensive ongoing research well documented by multiple publications up to date. ${ }^{16,22,23,24,25} \mathrm{~A}$ well known example is TRUMPF's TOP Cleave focusing optics (Figure 17) forming a Bessel-like beam where the USP laser is focused inside e.g. a Corning Gorilla ${ }^{\circledR}$ glass or even a sapphire to modify the material along the lengthened laser penetration line. Underlying the material modification by absorbing a vast amount of optical energy is the effect that high spatial and temporal photon density is getting absorbed even in transparent material due to nonlinear photon absorption processes generating free electrons which are causing absorption of additional photons. Depending on the pulse energy and the material properties, process speeds of more than $>1 \mathrm{~m} / \mathrm{s}$ for glass thicknesses $>3 \mathrm{~mm}$ in a single pass with volume modifications along the entire thickness of the substrate become feasible using adapted distributions of the focal field. This requires extreme aspect ratios of longitudinal (determined by the glass thickness) to transverse beam dimensions of $>1000: 1$ (see Figure 18). ${ }^{23,24}$ Those techniques can be further combined with sophisticated optical elements such as phase-only spatial light modulators (SLMs) in order to even cut glass samples with chamfers using aberration-corrected Bessel-like beams. ${ }^{27}$ The resulting absorption distribution and spatial energy deposition inside the material follows accurately the simulated intensity profile of the ultra-short pulsed laser beam as can be shown by using pump-probe microscopy. ${ }^{24,27}$

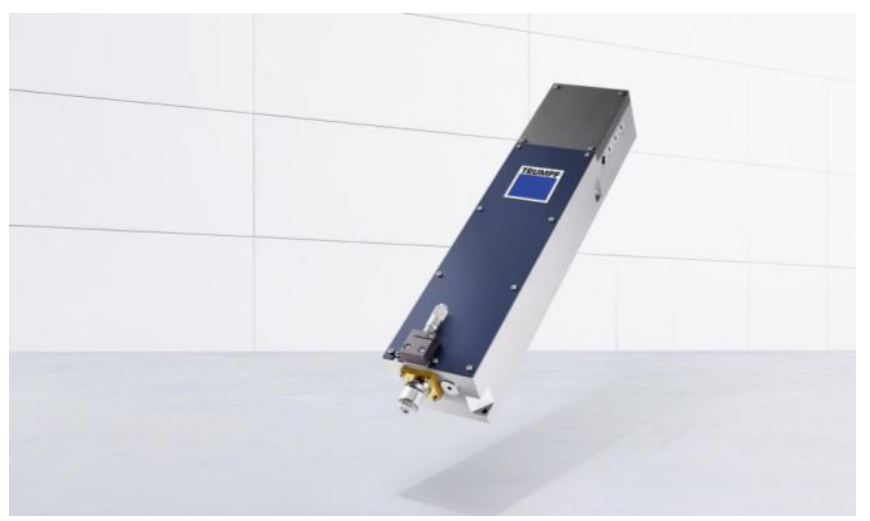

Figure 17. TOP Cleave optical head.

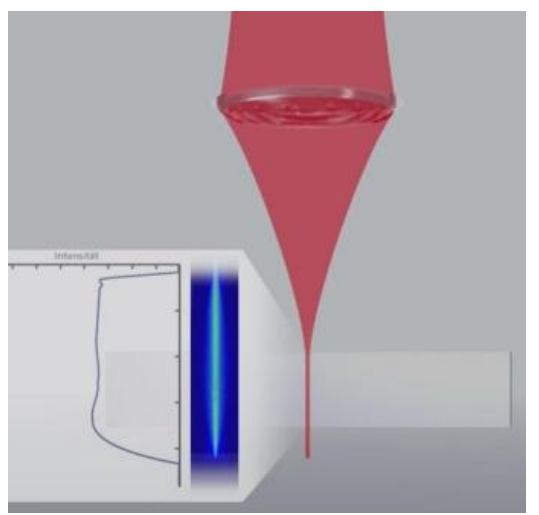

Figure 18. Distribution of the laser intensity in the lengthened focus along the beam axis. 
Shaping the laser beam and defining the process parameters appropriately offers further potential for applications like ultra-short pulse induced welding of fused silica without a previous optical contact of the two samples. ${ }^{23,25,26}$ A large pool of molten material $(<450 \mu \mathrm{m}$ long and $<160 \mu \mathrm{m}$ in diameter) can be reached using the previously mentioned TruMicro 2020 in burst mode (pulse energy $<10 \mu \mathrm{J}$; repetition rate $=200 \mathrm{kHz}$ ) to even bridge a gap of up to $3 \mu \mathrm{m}$ between fused silica samples as depicted in Figure 19A. ${ }^{25,26}$ Significant enhancement of the process stability has already been proven by implementing this process into TRUMPF's production system for multi-path welding (Figure 19B) of glass protection caps (Figure 19C) used for LLK-B laser light cables from TRUMPF made of fused silica (Figure 19D) ${ }^{28}$ Besides the economical advantages, the glass-welding process provides durable joint solution of high strength reaching about $85 \%$ of the stability of the pristine bulk material. ${ }^{25,28}$
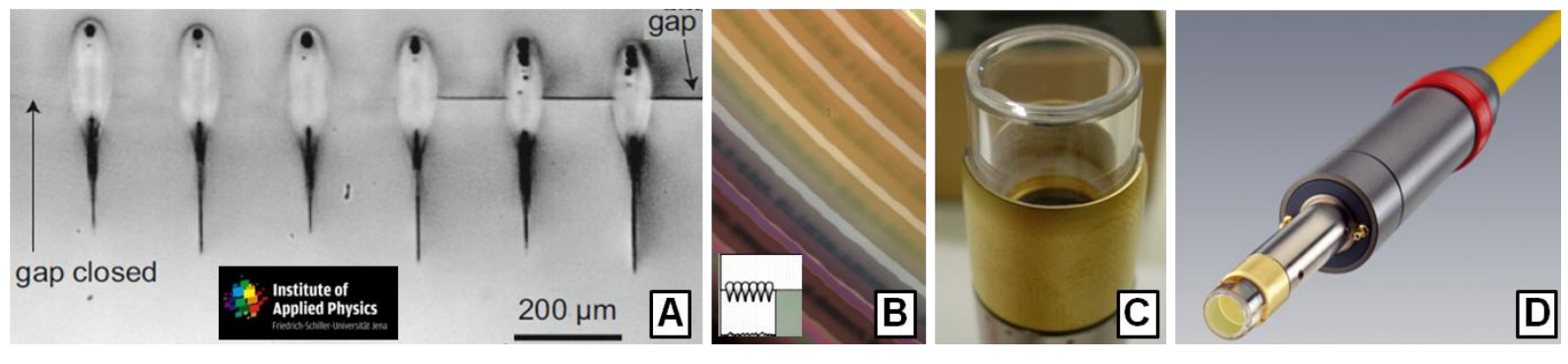

Figure 19. Ultra-short pulse induced welding of fused silica and its application to the production of TRUMPF's LLK-B laser light cables. ${ }^{26}$

In contrary, rapid thermal processing (RTP) of large area substrates with robust high-power lasers sources is very attractive due to the advantage of the approach to very locally deposit the energy required for the process. Consequently, applications such as tempering of multi-functional coatings, a lift-off process of thin layers from substrates used for the production of $\mathrm{OLEDs}^{29}$ or solid-state laser annealing of silicon for semiconductor manufacturing become feasible. ${ }^{30,31}$ For the latter, the laser beam induced melting and solidification of thin silicon films is used to attain a semi-crystalline grain structure of high uniformity as required for high-resolution thin-film transistor (TFT) displays with increased performance of the driver electronics for the pixels - as depicted in Figure 20. ${ }^{32}$

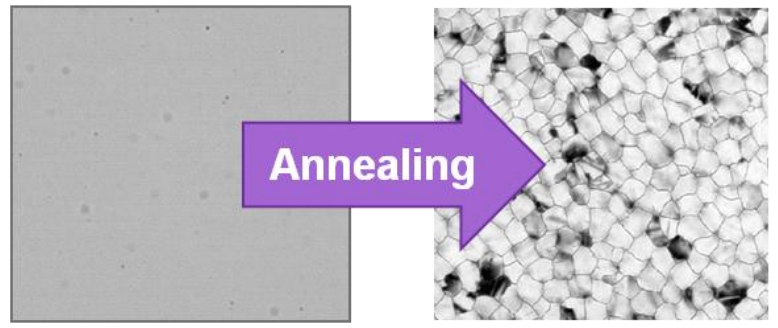

amorphous silicon $\rightarrow$ semi-crystalline silicon

Figure 20. Change of crystalline structure by means of SLA.

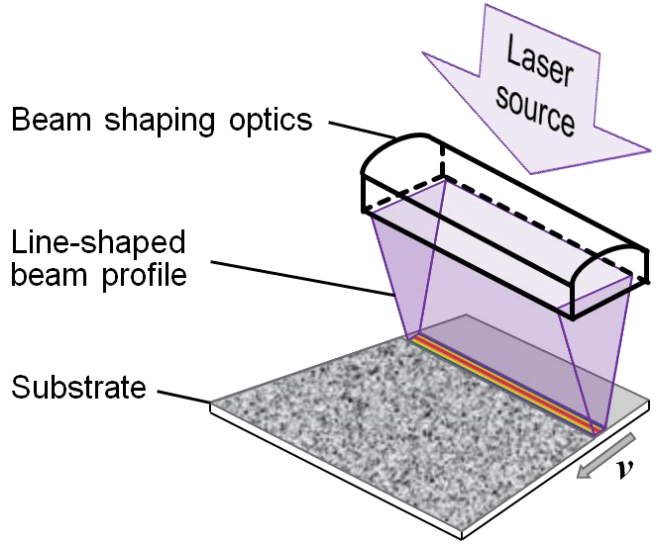

Figure 21. Sketch of laser-based rapid thermal processing of large-area substrates with linear scanning and lineshaped beam profile.

The optical concept of TRUMPF's line beam optics as schematically depicted in Figure 21 is unique with respect to its beam transformation and beam shaping technique, its efficiency and compactness and its multi-kW operational powerlevel. It provides reduced complexity, since the total number of modules is low compared to free-beam approaches. ${ }^{31}$ 


\section{SUMMARY AND OUTLOOK}

Supporting various technology platforms is central to TRUMPF's success in the world of industrial lasers. As new power hungry- applications in the field of micro-electronics arise, thin-disk laser become of increasing importance in the field of USP laser applications, while pulsed fiber laser systems find their applications more in the field of advanced emobility and in micro-welding applications e.g. of dissimilar materials. Long-term, we see various laser platforms existing in parallel at comparable cost levels. Depending on the field of application, the customer and the related special requirements each system has its preferred domain. Always keeping in mind the development of laser applications in industry has clearly not ended, yet.

\section{ACKNOWLEDGEMENT}

We gratefully acknowledge and want to thank all colleagues from TRUMPF, in particular Hagen Zimer, for their help, fruitful discussions and their valuable contributions.

\section{REFERENCES}

[1] Fies, A., "Milestones in the history of TRUMPF," Ditzingen, 25 January 2018, $<$ https://www.trumpf.com/en_US/company/trumpf-group/milestones-in-the-history-of-trumpf/1985-1995/> ( $2^{\text {nd }}$ January 2018).

[2] Leibinger, B., [Who Could Wish For Any Other Time But This - A Life Story], Murrmann Verlag GmbH, Hamburg, Germany (2011).

[3] Endo, A., "CO2 Laser Produced Tin Plasma Light Source as the Solution for EUV Lithography," [Lithography], InTech, Michael Wang (Ed.) (2010). ISBN: 978-953-307-064-3.

[4] Wissert, M., "TRUMPF laser amplifier for EUV lithography," Internal company presentation, Ditzingen (2016).

[5] Mayer, A., [High Power Laser Systems for Materials Processing], Optech Consulting, Taegerwilen, Switzerland (2015).

[6] Schad, S.-S., Kuhn, V., Gottwald, T., Negoita, V., Killi, A., Wallmeroth, K., "Near fundamental mode highpower thin-disk laser," Proc. SPIE 8959-31 (2014).

[7] Schad, S.-S., Gottwald, T., Kuhn, V., Ackermann, M., Bauer, D., Scharun, M., Killi, A., "Recent development of disk lasers at TRUMPF," Proc. SPIE 9726-41 (2016).

[8] Feuchtenbeiner, S., Zaske, S., Schad, S.-S., Gottwald, T., Kuhn, V., Kumkar, S., Killi, A., "New Generation of Compact High Power Disk Lasers," Proc. SPIE 10511-19 (2018).

[9] Speker, N., "BrightLine Weld - spatter-reduced high-speed laser-beam welding," Proc. European Automotive Laser Applications (EALA) (2017).

[10] Pantsar, H., Gabzdyl, J., Kaiser, E., Hesse, T., Kirchhoff, M., Faisst, B., New welding techniques and laser sources for battery welding," Proc. SPIE 10525-13 (2018).

[11] Ried, S., Rauch, S., Irmler, L., Rikels, J., Killi, A., Papastathopoulos, E., Sarailou, E., Zimer, H., "Next Generation Diode Lasers with Enhanced Brightness," Proc. SPIE 10514-15 (2018).

[12] Kahmann, M., Gebs, R., Fleischhaker, R., Zawischa, I., Kleinbauer, J., Russ, S., Bauer, L. Keller, U., Faisst, B. Budnicki, A., Sutter, D.,"Non-infrared femtosecond lasers: Status and prospects," Proc. SPIE 97400T (2016).

[13] Schad, S.-S., Stolzenburg, C., Michel, K. and Sutter, D., "Latest advances in high-brightness disk lasers high power performance from CW to short and ultra short pulses," in Laser Technik Journal, vol. 11, no. 2, pp. 49-53, April 2014.

[14] Stolzenburg, C., Schuele, W., Angrick, V., Montasser, B., and Killi, A., "Multi-kW IR and green nanosecond thin-disk lasers," Proc. SPIE 8959-25 (2014). 
[15] Heckl, O., Kleinbauer, J. Bauer, D., Weiler, S., Metzger, T., Sutter, D., "Ultrafast Thin-Disk Lasers", in Nolte, S. Schrempel, F., Dausinger, F. (Eds.), Ultrashort Pulse Laser Technology, Springer International Publishing, 2016. 3-22. (pp.93-115)

[16] Kumkar, M., "Beam shaping and in-situ diagnostics for development of transparent materials processing," Proc. SPIE 10522-15 (2018).

[17] Negel, J.-P., Loescher, A., Voss, A., Bauer, D., Sutter, D., Killi, A., Ahmed, M. A. and Graf, T.,"Ultrafast thin-disk multipass laser amplifier delivering $1.4 \mathrm{~kW}(4.7 \mathrm{~mJ}, 1030 \mathrm{~nm})$ average power converted to $820 \mathrm{~W}$ at $515 \mathrm{~nm}$ and $234 \mathrm{~W}$ at $343 \mathrm{~nm}$," Optics Express, vol. 23, no. 16, pp. 21064-21077 (2015).

[18] Teisset, C. Y., Wandt, C., Schultze, M., Klingebiel, S., Häfner, M., Prinz, S., Stark, S., Grebing, S., Negel, J.P., Höck, H., Scharun, M., Dietz, T., Bauer, D., Budnicki, A., Stolzenburg, C., Sutter, D., Killi, A., and Metzger, T.; "Multi-kW Thin-Disk Amplifiers", To be published in Proc. OSA High-Brightness Sources and Light-Driven Interactions Congress, Strasbourg, France (2018).

[19] Prinz, S., Schnitzenbaumer, M., Potamianos, D., Schultze, M., Stark, S., Häfner, M., Teisset, C. Y., Wandt, C., Michel, K., Kienberger, R., Bernhardt, B., Metzger, T., "Thin-disk pumped optical parametric chirped pulse amplifier delivering CEP-stable multi-mJ few-cycle pulses at $6 \mathrm{kHz}$," in Optics express, vol. 26, no. 2, pp. 1108-1124, January 2018.

[20] Wandt, C., Klingebiel, S., Schultze, M., Prinz, S., Teisset, C. Y., Stark, S., Grebing, C., Häfner, M., Bessing, R., Herzig, T., Budnicki, A., Sutter, D., Michel, K., Nubbemeyer, T., Krausz, F., Metzger, T., "1 kW Ultrafast Thin-Disk Amplifier System," Proc. CLEO, San Jose, San Jose, Ca, United States (2017).

[21] Michel, K., Klingebiel, S., Schultz, M., Teisset, C. Y., Bessing, R., Häfner, R., Prinz, S., Sutter, D., Metzger, T., "High-energy ultra-short pulse thin-disk lasers: new developments and applications," Proc. SPIE 972614 (2016).

[22] Kumkar, M., Kaiser, M., Kleiner, J., Flamm, D., Grossmann, D., Bergner, K., Zimmermann, F., and Nolte, S., "Throughput scaling by spatial beam shaping and dynamic focusing," Proc. SPIE 10091-15 (2017).

[23] Guertler, Y., Kahmann, M., Havrilla, D. L., "Beam shaping as an enabler for new applications," Proc. SPIE 10097-4 (2017).

[24] Flamm, D., Bergner,K., Grossmann, D., Hellstern, J., Kleiner, J., Jenne, M., Nolte, S., and Kumkar, M., "Higher-order Bessel-like beams for optimized ultrafast processing of transparent materials," Proc. CLEO/Europe-EQEC, Munich, Germany (2017)

[25] Richter, S., Zimmermann, F., Sutter, D., Budnicki, A., Tuennermann, A., and Nolte, S., "Ultrashort pulse laser welding of glasses without optical contacting," Proc. SPIE 10094-37 (2017).

[26] Richter, S., Zimmermann, F., Eberhardt, R., Tünnermann, A., Nolte, S., "Toward laser welding of glasses without optical contacting," in Applied Physics A, vol. 121, no. 1, pp 1-9, October 2015.

[27] Jenne, M., Flamm, D., Quaj, T., Hellstern, J., Kleiner, J., Grossmann, D., Kumkar, M., and Nolte, S., "Highquality glass chamfers using aberration-corrected Bessel-like beams," To be published (2018).

[28] Kaiser, E., "Laser welding of glass replaces glueing procedure," in Laser Technik Journal, vol. 13, no. 3, pp. 22-25, May 2016.

[29] Press Release, "Samsung Display replaces Excimer lasers with DPSS (Diode Pumping Solid-State) lasers for LLO," OLED Association, November 13, 2017, <http://www.oled-a.org/samsung-display-replaces-excimerlasers-with-dpss-diode-pumping-solid-state-lasers-for-llo-november-13-2017.html > (14 ${ }^{\text {th }}$ January 2018)

[30] Tillkorn, C., Canova, L., Dorer, S., Lang, M. and Huonker, M., "Novel optical concept for large area rapid thermal processing," Proc. Lasers in Manufacturing (2017).

[31] Tillkorn, C., Heimes, A., Flamm, D., Dorer, S., Hellstern, J., Lingel, C., Marschall, F., "Anamorphic beam shaping for efficient laser homogenization: Methods and high power applications," Proc. SPIE 10518-55 (2018).

[32] Im, J. S., "A new spot-beam-based laser-crystallization method for manufacturing advanced AMOLED displays," in SID Symposium Digest of Technical Papers, vol. 48, no. 1, pp. 418-419 (2017). 